\title{
Regional heterogeneity within the aorta: Relevance to aneurysm disease
}

Jean Marie Ruddy, MD, Jeffrey A. Jones, $\mathrm{PhD}$, Francis G. Spinale, MD, PhD, and John S. Ikonomidis, MD, $\mathrm{PhD}$

\begin{abstract}
Vascular remodeling within the aorta results in a loss of structural integrity with consequent aneurysm formation. This degradation is more common in the abdominal aorta but also occurs above the diaphragm in the thoracic aorta. Conventionally, the aorta has been considered a large vascular conduit with uniform cellular and extracellular structure and function. Evidence is accumulating, however, to suggest that variations exist between the thoracic and abdominal aorta, thereby demonstrating regional heterogeneity. Further pathophysiologic studies of aortic dilation in each of these regions have identified disparities in atherosclerotic plaque deposition, vessel mechanics, protease profiles, and cell-signaling pathways. Improved understanding of this spatial heterogeneity might promote evolution in the management of aneurysm disease through computational models of aortic wall stress, imaging of proteolytic activity, targeted pharmacologic treatment, and application of region-specific gene therapy.
\end{abstract}

Vascular remodeling within the aorta results in a loss of structural integrity with consequent aneurysm formation. This degradation is more common in the abdominal aorta but also occurs above the diaphragm in the thoracic aorta. Conservative management of small aneurysms has included serial imaging and pharmacologic treatment of associated risk factors while surgical intervention has been withheld until the risk of rupture exceeded that of repair. ${ }^{1}$ Despite these efforts, patients continue to experience significant morbidity and mortality from ruptured aneurysms, and therefore a great need exists for improved methods of risk stratification, prognosis prediction, and surgical decision making. ${ }^{2}$ Achieving this goal will require a fundamental understanding of aneurysm disease, integrating vascular physiology, cell biology, and vessel mechanics as an adjunct to the concept of aortic regional heterogeneity. The pathophysiology of the aorta above and below the diaphragm has demonstrated disparities in atherosclerotic susceptibility, vessel mechanics, proteolytic profiles, and cell-signaling pathways that have implications in the development of an aortic aneurysm. The objective of this review is to present literature describing the physical and molecular characteristics of the thoracic versus the abdominal aorta and provide evidence supporting the hypothesis that aortic regional heterogeneity exists. Additionally, we plan to delineate not only how these details influence aneurysm development but also their potential application in diverse, site-specific therapeutic strategies.

\footnotetext{
From the Division of Cardiothoracic Surgery, Department of Surgery, Medical University of South Carolina, Charleston, SC.

Supported by National Institutes of Health/National Heart, Lung, and Blood Institute grant R01-HL075488-04.

Received for publication May 27, 2008; accepted for publication June 24, 2008. Address for reprints: John S. Ikonomidis, MD, PhD, Division of Cardiothoracic Surgery, Department of Surgery, Medical University of South Carolina, 96 Jonathan Lucas St, Suite 409 CSB, PO Box 250612, Charleston, SC 29425 (E-mail: ikonomij@musc.edu).

J Thorac Cardiovasc Surg 2008;136:1123-30

$0022-5223 / \$ 34.00$

Copyright (C) 2008 by The American Association for Thoracic Surgery doi:10.1016/j.jtcvs.2008.06.027
}

\section{EPIDEMIOLOGIC HETEROGENEITY}

Aortic aneurysms remain the 13th most common cause of death in the United States, despite advances in screening programs, imaging, and endovascular interventions. ${ }^{1}$ Demographic studies have indicated that approximately $9 \%$ of the population older than 65 years have an abdominal aortic aneurysm (AAA), but thoracic aortic aneurysms (TAAs) are far less common, with a rate of 5.9 per 100,000 person-years. ${ }^{3,4}$ Five-year survival for TAAs has been approximated at only $64 \%,{ }^{1}$ whereas a randomized prospective trial of immediate repair versus watchful waiting of AAAs has reported a survival rate of $75 \%$ to $80 \%$ over the 8 -year study period. ${ }^{5}$ Men are more often affected than women in both aortic regions, and shared associated risk factors also include advanced age, cigarette smoking, hypertension, chronic obstructive pulmonary disease, and coronary artery disease. ${ }^{4,6}$ Aortic atherosclerotic plaque deposition, on the other hand, has been well documented in association with AAA growth, without a clear cause-and-effect mechanism identified at this time. ${ }^{7}$ Although some TAAs are associated with atherosclerotic disease, many occur in the complete absence of plaque deposition. ${ }^{7}$ Continued investigation into disparities among patient populations might help define TAA and AAA as unique disease entities.

The genetic contribution to aneurysm disease in the thoracic and abdominal aorta has been a prominent and well-studied variation. Approximately $20 \%$ of TAAs are attributed to some form of genetic syndrome. ${ }^{1}$ Connective tissue disorders, such as Marfan syndrome and EhlerDanlos syndrome type IV can affect any portion of the aorta but preferentially cause dilation of the thoracic aorta. ${ }^{1}$ Mutations of growth factor receptors have also been shown to predispose to TAA formation in both Loeys-Dietz syndrome and familial TAA and dissection syndrome. ${ }^{8,9}$ Additionally, the common congenital anomaly of bicuspid aortic valve has been associated with TAA growth. ${ }^{10}$ In AAA, on the other hand, the genetic predisposition reported in $12 \%$ to $19 \%$ of patients with AAA has not yet been traced to particular mutations but coincides with a family history of a firstdegree relative with aneurysm disease. ${ }^{11}$ The varying 
contributions of genetic and environmental influences on TAA versus AAA development support the premise that the 2 are unique pathophysiologic entities.

\section{EMBRYOLOGIC HETEROGENEITY}

Heterogeneity between the thoracic and abdominal aortic regions begins during embryogenesis. ${ }^{12}$ Neural crest cell precursors to the thoracic aorta and mesodermal ancestors of the abdominal aorta have demonstrated unique responses to various cytokines and growth factors. For instance, homocysteine, a nonprotein amino acid that in increased levels conveys a predisposition for cardiovascular disease, has been shown to stimulate proliferation of neural crestderived vascular smooth muscle cells (SMCs), with no effect on mesodermal SMCs. ${ }^{13}$ Also, collagen precursor production is increased in neural crest, but not mesodermal, SMCs after exposure to transforming growth factor $\beta$ (TGF- $\beta$ ). ${ }^{12}$ In addition, altered DNA synthesis ${ }^{12}$ and contractile response have been reported. ${ }^{14}$ These distinct and diverse reactions to individual stimuli, based solely on embryologic origin, have highlighted the potential disparities in aortic wall behavior observed above and below the diaphragm, the end result of which might manifest as aneurysm disease.

\section{STRUCTURAL HETEROGENEITY}

The aorta is a large elastic artery composed of 3 layers: the intima, media, and adventitia. A single layer of endothelial cells sitting on loose connective tissue comprises the intima, the media consists of SMCs embedded in a dense matrix of fibrillar structural proteins, and the adventitia contains fibroblasts and collagen fibers. In the thoracic, as well as abdominal, segments of the aorta, aneurysm formation is attributed to degradative remodeling of the medial extracellular matrix (ECM) and SMC loss, ${ }^{2}$ and therefore this layer of the aortic wall will be discussed in greater detail.

The aortic media provides viscoelasticity through concentric bands of elastin filaments with associated collagen fibers and SMCs, which are termed lamellar units. ${ }^{15}$ In the mammalian aortic media the first 28 to 30 lamellar units from the luminal surface do not contain a blood supply, thereby forming an avascular zone that receives oxygen and nutrients by means of transintimal diffusion from the plasma. ${ }^{15}$ When additional units are present, vasa vasorum from the adventitia penetrate the outer media, creating a vascular zone. ${ }^{15}$ This principle remains true in human subjects and provides a clear distinction between the thoracic and abdominal aorta. The thoracic aorta contains 55 to 60 lamellar units divided into vascular and avascular zones, whereas the entirely avascular abdominal aortic media typically contains 28 to 32 units. ${ }^{15}$ Differences in oxygen, nutrient, and growth factor delivery to the cells of the thoracic and abdominal aortic media likely contribute to the variations identified in vascular remodeling.
Interestingly, the media has also been shown to grow differently above and below the diaphragm. Medial thickness increases from birth to adulthood, and in the thoracic aorta additional lamellar units are synthesized, whereas this expansion occurs by widening each unit of the abdominal aorta. ${ }^{16}$ The assembly of additional lamellar units might explain the increased elastin content of the thoracic aorta. ${ }^{17}$ Both of these growth mechanisms, however, maintain a constant ratio of aortic diameter to medial thickness, influencing both wall stress and tension per lamellar unit, as discussed in the subsequent section. ${ }^{16}$ Aneurysm disease involves elastin fragmentation and SMC death, and therefore an aortic region with fewer lamellar units, decreased elastin content, and poorer nutrient delivery to the SMCs, such as the abdominal aorta, might be at considerably increased risk of medial degeneration and aneurysm formation.

\section{MECHANICAL HETEROGENEITY}

The ultimate goal in the care of a patient with an aortic aneurysm is to prevent rupture, a material failure that occurs when the aortic wall stress exceeds the tensile strength. ${ }^{18}$ The material properties of the normal and aneurysmal aorta with regard to breaking stress and distensibility or stiffness have been an area of intense study. According to the law of LaPlace, wall tension is influenced by intraluminal pressure, vessel diameter, and wall thickness, and the application of this tension to a defined area indicates wall stress. ${ }^{19} \mathrm{~A}$ consistent ratio of aortic diameter to medial thickness is maintained throughout all mammalian species, including the human thoracic and abdominal aortas. ${ }^{16}$ The proportional change in both aortic diameter and wall thickness observed above versus below the diaphragm therefore leads to uniform wall stress in the thoracic and abdominal aortas under physiologic conditions. ${ }^{16}$ As the phrase implies, the wall stress at which rupture occurs can be called the breaking stress, ${ }^{20}$ and similar values for breaking stress ranging from $172 \pm 90 \mathrm{~N} / \mathrm{cm}^{2}$ to $270 \pm 150 \mathrm{~N} / \mathrm{cm}^{2}$ have been recorded in healthy thoracic and abdominal aortic regions. ${ }^{20,21}$ All tissue specimens harvested from aneurysms have had reduced breaking stress, but the strength of TAA samples was roughly twice that of AAA samples $\left(121 \pm 33 \mathrm{~N} / \mathrm{cm}^{2}\right.$ vs 65 $\pm 9 \mathrm{~N} / \mathrm{cm}^{2}$, respectively), ${ }^{18,22}$ underscoring the mechanical heterogeneity of these 2 regions.

Additionally, the abdominal aorta has fewer lamellar units, and therefore the same wall stress absorbed by the thoracic aorta is dispersed across fewer units, leading to increased wall tension per lamellar unit ${ }^{16}$ and subsequently increased tension per SMC. Many studies have demonstrated that mechanical forces can alter SMC transcriptional activity to influence matrix structure, as well as cell survival. ${ }^{23}$ This link between mechanical force and SMC synthetic activity has a potential role not only in defining the apparent increased susceptibility of the abdominal aorta but also in aneurysm initiation in general. 
A change in aortic radius caused by an incremental change in pressure describes vessel distensibility, and a lack of distensibility or resistance to deformation is referred to as stiffness. ${ }^{20,22}$ The reduced elastin content quantified in the abdominal aorta ${ }^{17}$ might explain the increased stiffness documented in this region at $569 \pm 138 \mathrm{~N} / \mathrm{cm}^{2}$ as opposed to $261 \pm 26 \mathrm{~N} / \mathrm{cm}^{2}$ in the thoracic aorta. ${ }^{22,24}$ Additionally, studies comparing normal and aneurysmal aortic tissues have consistently demonstrated increased stiffness in both TAA and AAA specimens, potentially because of further loss of elastin fibers and accumulation of disorganized collagen. ${ }^{18,22,24}$ Continued investigation to identify heterogeneity in the material and mechanical properties of the walls of TAAs versus AAAs might lead to differential prognostic profiles for these disease processes.

The stiffness of the abdominal aorta and reduced breaking stress of the AAA wall has proved useful in aneurysm monitoring. The unique material characteristics of the abdominal aorta have been used, along with patient parameters and 3-dimensional imaging, to formulate mathematic models of wall stress and tensile strength. Current work in AAAs has demonstrated improved ability to predict rupture compared with the simplified law of LaPlace and the maximal size criterion, and further application to TAAs is warranted. ${ }^{19}$ As these mathematic approximations of aortic wall stress continue to become more precise and the software progressively evolves into a marketable format, surgical intervention will rely on serial wall-stress measurements until a known high-risk tension threshold is approached. This value would likely vary by age, sex, and race in addition to aneurysm location. Further coupling of imaging techniques to quantify proteolytic activity in conjunction with wall stress could provide the surgeon with a comprehensive profile of each individual aneurysm and allow for truly patient-specific surgical decision making.

\section{HETEROGENEITY OF ATHEROSCLEROTIC PLAQUE DEPOSITION}

Atherosclerosis is a chronic progressive disease of large elastic and muscular arteries involving lipid deposition, collagen production, and inflammatory cell infiltration, ${ }^{25}$ the biochemical and molecular scope of which exceeds the focus of this review. The disease, however, affects the aorta differently above and below the diaphragm and has been closely associated with aneurysm formation, and therefore the anatomic and histologic features of aortic atherosclerotic disease will be outlined to support the principle of regional heterogeneity within this vessel. The Pathological Determinants of Atherosclerosis in Youth study reported an increased incidence of atherosclerotic lesions in the abdominal aorta. ${ }^{25}$ The thoracic aorta appears more resistant to plaque formation, and has demonstrated a low likelihood of fatty streak progression. ${ }^{26}$ The fatty streaks identified in the abdominal aorta, on the other hand, represent early athero- sclerotic lesions and have shown consistent progression to high-grade lesions over the ensuing 10 years, presumably because of intimal thickening and increased collagen production. ${ }^{26}$ The preferential growth of atherosclerotic plaque in the abdominal aorta has been attributed to variations in flow and shear stress in that region. ${ }^{25}$ Differing susceptibility to lipid deposition and subsequent plaque evolution also suggests disparate cellular and extracellular composition between the thoracic and abdominal aortas. Consequently, the influence of atherosclerotic plaque on aneurysmal dilation of the abdominal aorta would not be present in the thoracic aorta, supporting a separate pathophysiologic mechanism for TAA development. Further investigation into the link between atherosclerosis and aneurysm disease might concomitantly provide evidence corroborating aortic regional heterogeneity.

\section{HETEROGENEITY WITHIN PROTEINASE SYSTEMS}

The role of proteolysis in cardiovascular disease, including atherosclerosis, restenosis, thrombosis, and aneurysm formation, has been well documented. ${ }^{27}$ In particular, aneurysm formation has been attributed to lysis of elastin and collagen in the aortic media, thereby weakening the vessel wall. $^{2}$ The matrix metalloproteinases (MMPs), a family of major ECM-remodeling enzymes, are responsible for angiogenesis, wound healing, and cardiomyopathy. ${ }^{27}$ MMPs and their antagonists, the tissue inhibitors of metalloproteinases (TIMPs), are instrumental in aneurysm disease. ${ }^{27} \mathrm{~A}$ comprehensive understanding of the activity of these enzymes in thoracic versus abdominal aneurysm disease might define molecular diversity within the aortic wall and identify divergent pathways for novel, site-specific therapeutic interventions.

\section{MMPs}

MMPs are divided into subclasses based on substrate specificity, such as gelatinases, elastases, and collagenases. ${ }^{27}$ Members of the gelatinase subclass, MMP-2 and MMP-9, have demonstrated an ability to degrade denatured fibrillar collagen (gelatin), elastin, and native types IV, V, and VII collagen, along with other ECM components. ${ }^{28} \mathrm{MMP}-2$, or gelatinase A, is constitutively expressed by SMCs of the aortic media and also displays interstitial collagenase capabilities, cleaving type I collagen, which comprises approximately $60 \%$ of the collagen content of the aorta. ${ }^{28}$ MMP- 9 is also referred to as gelatinase B and can be produced by macrophages, fibroblasts, or SMCs that have been stimulated to undergo a phenotypic switch favoring synthetic activity. ${ }^{29}$ The gelatinases have been heavily studied with regard to aneurysm formation in both the thoracic and abdominal aortas.

The contribution of MMP-2 to TAAs has been difficult to define. Studies focusing on idiopathic TAAs have failed to show an increase in MMP-2 levels compared with those 
seen in normal aortas. ${ }^{30}$ TAAs associated with atherosclerosis, on the other hand, have demonstrated increased MMP-2 activity. ${ }^{31}$ Different biochemical tests were used to quantify MMP-2 mRNA versus active protease levels versus latent protease levels, making comparisons between studies difficult. Also, increasing age alone has been associated with increased MMP-2 levels in the thoracic aorta, and therefore the use of age-matched control subjects might have obscured the effect of MMP-2 on TAAs. ${ }^{32}$ A murine model of TAA development has reported increases in MMP-2 levels as early as 72 hours after aneurysm induction, with return to baseline by 2 weeks, ${ }^{33}$ suggesting a role for MMP-2 in early TAA growth that perhaps has not been captured in human tissue samples harvested at the time of aneurysm repair.

In AAAs the data are more definitive that MMP-2 is instrumental to aneurysm development. Tissue samples harvested at the time of aneurysm repair have consistently shown increased MMP-2 expression and activity caused by amplified production by native SMCs. ${ }^{34,35}$ More interestingly, Freestone and colleagues ${ }^{35}$ demonstrated that aneurysms smaller than $5.5 \mathrm{~cm}$ had greater MMP-2 activity than larger aneurysms and proposed that early AAA growth is directed by MMP-2. The vital role of MMP-2 in AAA formation has been corroborated in a murine model of AAA in which aneurysms could not be induced in MMP-2 knockout mice. $^{36}$

The observation that increased MMP-2 activity is required for AAA growth but might or might not influence TAA development represents an emerging concept in aortic heterogeneity. The differential contribution of this proteinase to aneurysm disease appears to vary above and below the diaphragm, as well as within the thoracic aorta. Differences in MMP-2 levels have been reported in ascending versus descending TAAs, a finding that strengthens the argument for regional heterogeneity in aortic MMP production but has not been sufficiently explored to draw any firm conclusions. Additionally, variations in TAA MMP-2 levels have been related to sample location because most specimens are harvested from the anterior wall, but evidence has suggested that greater MMP-2 levels can be measured in the posterior wall of the TAA. ${ }^{30}$ The inflammatory infiltrate accompanying atherosclerotic plaque deposition might increase MMP-2 transcriptional activity in AAAs and atherosclerotic TAAs. ${ }^{31}$ Nonatherosclerotic TAAs, on the other hand, seem to be driven by different forces, and their effect on thoracic aorta SMC gene expression does not include upregulation of MMP-2 production, supporting the premise that multiple pathophysiologic pathways can lead to aneurysm formation.

In contrast to MMP-2, MMP-9 has demonstrated a key role in TAA disease. Increased MMP-9 levels have been reported in TAAs both with and without associated atherosclerosis. ${ }^{31}$ Murine TAA models have demonstrated attenuated aneurysm growth in MMP-9 knockout mice at 4 weeks after induction and MMP-9 abundance in wild-type mice beginning 2 weeks after induction. ${ }^{33,37}$ MMP-9 levels remained increased throughout aneurysm growth. ${ }^{33,37}$ Interestingly, immunohistochemical staining has localized MMP-9 to mesenchymal cells of the aortic media and adventitia, specifically fibroblasts, myofibroblasts, and SMCs that have undergone a phenotypic switch from purely contractile to synthetically active. ${ }^{37}$ In the healthy aorta these cells do not produce MMP-9, and such a shift in transcriptional activity has previously been attributed to alterations in cell-cell and cell-matrix interactions, ${ }^{29}$ suggesting that ECM remodeling might precede and actually help initiate MMP-9 production in the TAA.

MMP-9 is considered the primary elastolytic enzyme within the AAA wall and is credited with accelerated aneurysm progression. Markedly increased levels of MMP-9 have been consistently documented in AAA tissue and attributed to macrophages infiltrating the aortic media and adventitia. $^{36,38}$ Trends in MMP-9 expression have also been identified with regard to aneurysm size, such that aneurysms between 5 and $7 \mathrm{~cm}$ in diameter have more MMP-9 than those either larger or smaller. ${ }^{39}$ This evidence suggests a role for MMP-9 in aneurysm progression, perhaps to a point at which additional proteases or hemodynamic factors become the driving force in AAA growth and rupture. ${ }^{39}$ The importance of MMP-9 in AAA development has been further supported in a murine model in which aneurysm induction was inhibited in MMP-9 knockout mice. ${ }^{36}$ Confirmation of the macrophage as the primary source of MMP-9 in AAA was achieved when MMP-9 knockout mice were infused with wild-type macrophages and subsequent restoration of AAA growth was documented. ${ }^{36}$

The different cellular sources of MMP-9 in TAAs versus AAAs have substantiated a major point in the argument favoring aortic regional heterogeneity with regard to aneurysm disease. A hypothesis of AAA formation can be constructed from the prevalence of atherosclerotic plaque leading to macrophage infiltration, with consequent MMP-9 production and surrounding matrix degradation. In contrast, MMP-9 expression by native mesenchymal cells of the TAA and the low incidence of inflammatory infiltrate suggest that additional forces are acting in the thoracic aorta.

\section{TIMPs}

In addition to regulating MMP production and activity, factors stimulating aortic medial degeneration also affect the expression of TIMPs, the primary endogenous antagonists of MMPs. Aortic SMCs constitutively express TIMP-1 and TIMP-2, and TIMP-1 has been demonstrated to inhibit most MMPs, especially MMP-9, ${ }^{27}$ but the function of TIMP-2 is more complex. Although it can inhibit MMP-2 and MMP-9, TIMP-2 is also a component of the MMP-2 activation complex. ${ }^{40}$ The specific relationship between TIMPs and aneurysm disease has not yet been defined, 
but variability between TAAs and AAAs has become apparent.

The interplay of MMPs and TIMPs directs ECM turnover in healthy tissues, and the degradation associated with aneurysm growth in any location can be expected to stem from an alteration favoring proteolysis. More specifically, the ratio of MMP-9 to TIMP-1 has been used to estimate the proteolytic index of the ECM. ${ }^{38}$ In TAAs TIMP-1 levels have been decreased or unchanged from healthy aortic tissue, and therefore coupled with the increased MMP-9 levels, a large shift in the proteolytic state favoring ECM degeneration has been noted. ${ }^{31,41}$ AAA tissue samples, on the other hand, have demonstrated increased levels of TIMP-1 compared with those seen in control aortic specimens. ${ }^{38}$ Despite this amplified TIMP-1 production, the MMP-9/TIMP-1 ratio has favored proteolysis in AAAs because MMP-9 levels have been increased to a much greater extent. ${ }^{38}$ The differential role of TIMP-1 in this proteolytic shift supports the concept of disparate stimuli and mechanisms for aortic dilation in the thoracic and abdominal aortas.

The contribution of TIMP-2 to aneurysm development has been obscured by its dual role in activating, as well as inhibiting, MMP-2. ${ }^{40}$ Additionally, no consistent trend in TIMP-2 expression has been identified in either TAA or AAA samples, further complicating the issue. ${ }^{31,38,41}$ This variability can be largely attributed to the assorted biochemical techniques used to quantify TIMP-2 expression, abundance, or activity in each report. A few studies have evaluated a second proteolytic index defined as the ratio of MMP-2 to TIMP-2. ${ }^{31,34,38}$ In TAA specimens an increase in the MMP-2/TIMP-2 ratio has been observed, ${ }^{31}$ whereas that of AAA samples has remained unchanged from ratios seen in control aortic tissue. ${ }^{38}$ Therefore pertaining to TIMP-2, the SMC transcriptional response to aneurysminitiating stimuli has appeared to differ in the thoracic and abdominal aortas, potentially because of alternate causative factors, cell signaling, or proteolytic substrates.

Ideally, the identification and definition of proteolytic variations between the TAA and the AAA will allow sitespecific, minimally invasive interventions directed at slowing or halting aneurysm growth. General MMP inhibition is currently being explored, ${ }^{27}$ but the use of gene therapy through systemic introduction or regional placement with endostents to preferentially affect particular MMPs might narrow the potential side effect profile. For instance, targeting MMP-2 activity in small AAAs versus MMP-9 in advanced disease might be a useful stratification. In TAAs, on the other hand, MMP-9 inhibition could be beneficial, despite the degree of aortic dilation. More importantly, methods of quantifying MMP or TIMP activity might prove useful in surgical planning. Technetium-tagged small molecules that preferentially enter the vascular media and bind MMPs can be coupled with nuclear imaging to identify patients with increased proteolytic activity, signifying a propensity for rapid expansion and rupture. These patients might warrant urgent repair, despite their current aneurysm diameter. As mentioned earlier, the combination of computational stress analysis and proteolytic imaging might revolutionize aneurysm management.

\section{HETEROGENEITY IN INTERCELLULAR SIGNALING PATHWAYS}

Many investigators have focused on characterizing the protease systems responsible for aortic medial degeneration, and the signaling pathways initiating protease production remain poorly understood. The regulation of aortic SMC gene expression through cytokines and growth factors has been suspected to be a major contributor to aneurysm growth. ${ }^{2}$ Cytokines are intercellular mediators that direct the immune response to various stimuli, and a subfamily of cytokines, the chemokines, specifically act as potent chemoattractants and activators of leukocytes. The peptide growth factor TGF- $\beta$ encompasses a large family of messengers implicated in numerous cellular pathways, including angiogenesis, apoptosis, and tissue fibrosis. ${ }^{42}$ This growth factor can also maintain normal blood vessel morphology through stimulation of ECM production and inhibition of inflammatory mediators. ${ }^{42}$ Although not fully explored, current evidence suggests that the signaling pathways driven by cytokines and TGF- $\beta$ differentially affect aneurysm growth in the thoracic and abdominal aorta.

\section{IMMUNE MEDIATORS}

The presence and nature of the aneurysm wall inflammatory infiltrate can be considered a major defining factor in the argument favoring regional aortic heterogeneity. Levels of certain cytokines, such as tumor necrosis factor $\alpha$, interleukin $1 \beta$, and interleukin 6 , have been universally increased in both TAA and AAA tissue samples, ${ }^{43,44}$ presumably related to previous evidence that these cytokines augment MMP expression. ${ }^{45}$ The inflammatory infiltrate described in the media and adventitia of aneurysm walls has been dominated by $\mathrm{CD}^{+}{ }^{+} \mathrm{T}$-helper lymphocytes and macrophages. ${ }^{44}$ These lymphocytes can produce a proinflammatory or anti-inflammatory cytokine profile, classifying themselves as $T_{H} 1$ or $\mathrm{T}_{\mathrm{H}} 2$ cells, respectively, and whether one subgroup preferentially drives medial degeneration has important implications. In a TAA the presence of an inflammatory infiltrate has been a controversial issue. One particularly influential study examining TAA tissue described categories of infiltrated versus noninfiltrated aneurysms and further reported $\mathrm{a} \mathrm{T}_{\mathrm{H}} 1$-driven immune response. ${ }^{46}$ The increased levels of interferon $\gamma$ correlated with aneurysm diameter, intimal thickness, and elastin fragmentation. ${ }^{46}$ AAA specimens have demonstrated increased expression of many proinflammatory and anti-inflammatory cytokines, and studies aimed at defining the lymphocytic infiltrate as predominantly $\mathrm{T}_{\mathrm{H}} 1$ or $\mathrm{T}_{\mathrm{H}} 2$ have found evidence of both. ${ }^{47,48}$ Collectively, the 
TABLE 1. Summary of heterogeneity between the thoracic and abdominal aorta

\begin{tabular}{|c|c|c|c|}
\hline Variable & Thoracic aorta & Abdominal aorta & References \\
\hline \multirow[t]{2}{*}{ Epidemiology } & Rare aneurysm site & Most common aneurysm site & $1-11$ \\
\hline & $20 \%$ caused by genetic syndromes & $20 \%$ have familial predisposition & \\
\hline Embryology & Derived from neural crest & Derived from mesoderm & $12-14$ \\
\hline \multirow[t]{4}{*}{ Structure } & Vascular outer media & Avascular medial layer & $15-17$ \\
\hline & More numerous lamellar units & Fewer lamellar units & \\
\hline & Grows by synthesizing additional lamellar units & Grows by increasing lamellar unit thickness & \\
\hline & Greater elastin and collagen content & Lower elastin and collagen content & \\
\hline \multirow[t]{3}{*}{ Mechanics } & Greater distensibility & Increased stiffness & $18-24$ \\
\hline & $\begin{array}{l}\text { TAA breaking stress greater than AAA breaking } \\
\text { stress }\end{array}$ & $\begin{array}{l}\text { AAA breaking stress less than TAA breaking } \\
\text { stress }\end{array}$ & \\
\hline & & Increased tension per lamellar unit & \\
\hline \multirow[t]{2}{*}{ Atherosclerosis } & $\begin{array}{l}\text { Low likelihood of lesion progression from fatty } \\
\text { streak to atheroma }\end{array}$ & Site of most severe atherosclerosis & $25-26$ \\
\hline & & $\begin{array}{l}\text { High likelihood of lesion progression from fatty } \\
\text { streak to atheroma }\end{array}$ & \\
\hline \multirow[t]{4}{*}{ MMPs } & Inconsistent role for MMP-2 & Early aneurysm growth driven by MMP-2 & $27-39$ \\
\hline & $\begin{array}{l}\text { MMP-9 produced by synthetically active SMCs } \\
\text { and fibroblasts }\end{array}$ & MMP-9 produced by macrophages & \\
\hline & $\begin{array}{l}\text { Lack of MMP-9 attenuated aneurysm } \\
\text { development }\end{array}$ & MMP-9 proportional to aneurysm diameter & \\
\hline & & $\begin{array}{l}\text { Lack of MMP-9 prevented aneurysm } \\
\text { development }\end{array}$ & \\
\hline \multirow[t]{2}{*}{ TIMPs } & No change or decreased TIMP-1 & Increased TIMP-1 & $27-41$ \\
\hline & MMP-2/TIMP-2 ratio increased & MMP-2/TIMP-2 ratio unchanged & \\
\hline Immune mediators & $\begin{array}{l}\mathrm{T}_{\mathrm{H}} 1 \text {-type immune response in infiltrated } \\
\text { aneurysms }\end{array}$ & $\begin{array}{l}\text { Evidence of both proinflammatory and } \\
\text { anti-inflammatory cytokines }\end{array}$ & $43-48$ \\
\hline TGF- $\beta$ response & $\begin{array}{l}\text { Increased signaling contributes to aneurysm } \\
\text { disease }\end{array}$ & Overexpression attenuated proteolytic state & $49-50$ \\
\hline
\end{tabular}

data on immunologic mediators have suggested that the initiation and growth of an infiltrated TAA is driven by proinflammatory cytokines, whereas AAA development involves a complex interaction between opposing immunologic pathways, ultimately supporting heterogeneity in the pathophysiology of aortic aneurysm formation.

\section{TGF- $\beta$}

Alterations in TGF- $\beta$ expression, activation, or receptor activity might provide pathways toward aortic wall degeneration. TGF- $\beta$ is associated with the ECM microfibrils in the thoracic and abdominal aortas, and the concentration of this ligand has demonstrated tight regulation. ${ }^{49}$ A murine model of Marfan syndrome has described increased levels of biologically active TGF- $\beta$ in these animals and a correlation to TAA growth. ${ }^{49}$ Additionally, TGF- $\beta$ receptor mutations have been identified as the cause of the familial TAA and dissection and Loeys-Dietz syndromes, in which currently unidentified mechanisms have allowed enhanced TGF- $\beta$ signaling and TAA formation. ${ }^{8,9}$ In the abdominal aorta the opposite might be true. Evidence has suggested that enzymatic activity of MMP-2 and MMP-9 release TGF- $\beta,{ }^{49}$ the overexpression of which can then decrease gelatinase and increase TIMP production, potentially attenuating aneurysm development. ${ }^{50}$ Although not fully characterized, the role of TGF- $\beta$ in TAAs versus AAAs has demonstrated divergence, likely because of the disparate embryologic origins of the thoracic and abdominal aortas discussed earlier, and this represents yet another example of heterogeneity in aneurysm disease.

Modification of upstream signaling pathways might represent a viable approach to disrupting aneurysm growth. AAAs appear to be influenced by both proinflammatory and anti-inflammatory mediators, whereas a subset of TAAs are driven solely by cytokines released from $\mathrm{T}_{\mathrm{H}} 1$ type lymphocytes. Additionally, TGF- $\beta$ overexpression in AAAs and inhibition in TAAs might halt aneurysm progression. Systemic upregulation or blockade of these messengers would likely be detrimental to a patient's overall health, and therefore the use of directed gene therapy or regional application would be required. A rodent AAA model involved endostent-delivered gene therapy with TGF- $\beta$ and demonstrated a halt in aneurysm growth. ${ }^{50}$ Similarly, delivered blockade of interferon $\gamma$ in infiltrated TAAs might also have the potential to alter the extracellular milieu and stabilize the aortic wall. Further defining the molecular variances 


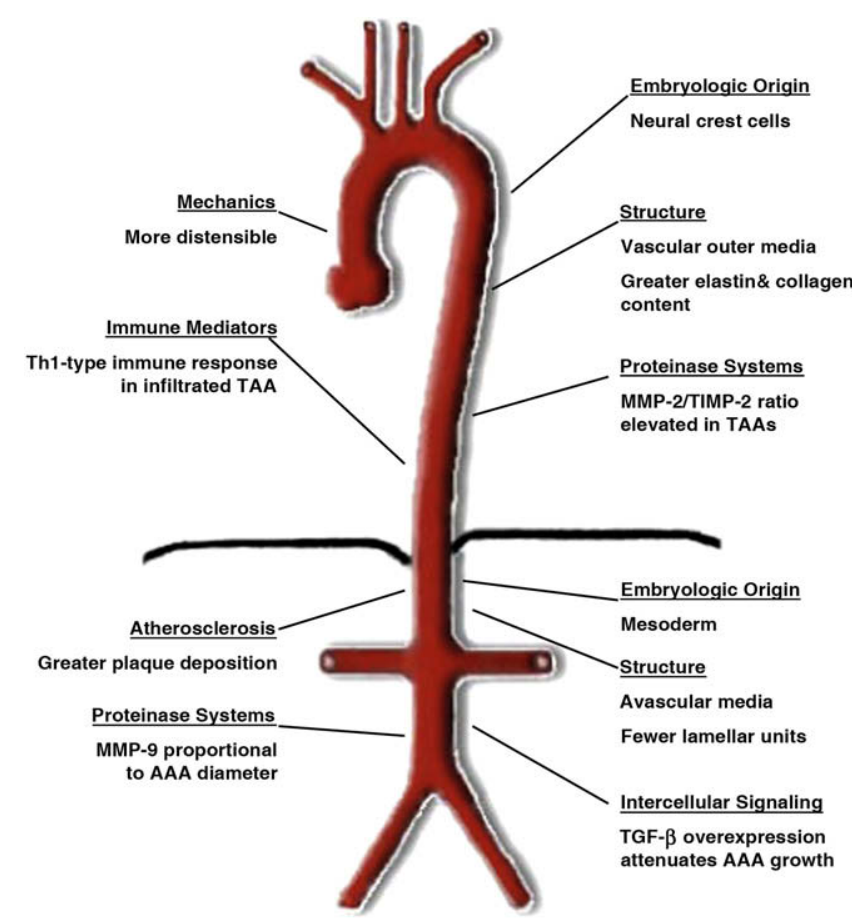

FIGURE 1. Pertinent disparities between the thoracic and abdominal aorta. $T A A$, Thoracic aortic aneurysm; $M M P$, matrix metalloproteinase; $T I M P$, tissue inhibitor of metalloproteinase; $A A A$, abdominal aortic aneurysm; $T G F-\beta$, transforming growth factor $\beta$.

among TAA and AAA could aide in the development of target-specific and region-specific minimally invasive aneurysm therapy.

\section{CONCLUSIONS}

An array of genetic, anatomic, biochemical, and mechanical factors have been recognized as contributors to aortic aneurysm disease, and a comprehensive model incorporating all these forces has yet to emerge. The hypothesis that heterogeneity exists within the aorta further complicates the study of this disease process. Variations among vessel mechanics, atherosclerotic plaque deposition, protease profiles, and cell-signaling pathways have been identified (Table 1). This review has sought to define and explore disparate properties in the thoracic and abdominal aortic segments as they pertain to aneurysm development (Figure 1) and furthermore to suggest how these variations can be exploited to advance current management of aortic aneurysm disease. Additional research into these regional distinctions is expected to uncover novel dynamic imaging and therapeutic options.

\section{References}

1. Coady MA, Rizzo JA, Goldstein LJ, Elefteriades JA. Natural history, pathogenesis, and etiology of thoracic aortic aneurysms and dissections. Cardiol Clin. 1999;17:615-35, vii.
2. Wassef M, Baxter BT, Chisholm RL, et al. Pathogenesis of abdominal aortic aneurysms: a multidisciplinary research program supported by the National Heart, Lung, and Blood Institute. J Vasc Surg. 2001;34:730-8.

3. Bickerstaff LK, Pairolero PC, Hollier LH, et al. Thoracic aortic aneurysms: a population-based study. Surgery. 1982;92:1103-8.

4. Lederle FA, Johnson GR, Wilson SE, et al. Prevalence and associations of abdominal aortic aneurysm detected through screening. Aneurysm Detection and Management (ADAM) Veterans Affairs Cooperative Study Group. Ann Intern Med. 1997;126:441-9.

5. Lederle FA, Wilson SE, Johnson GR, et al. Immediate repair compared with surveillance of small abdominal aortic aneurysms. N Engl J Med. 2002;346:1437-44.

6. Davies RR, Gallo A, Coady MA, et al. Novel measurement of relative aortic size predicts rupture of thoracic aortic aneurysms. Ann Thorac Surg. 2006;81:169-77.

7. Ito S, Akutsu K, Tamori Y, et al. Differences in atherosclerotic profiles between patients with thoracic and abdominal aortic aneurysms. Am J Cardiol. 2008;101: 696-9.

8. Loeys BL, Chen J, Neptune ER, et al. A syndrome of altered cardiovascular, craniofacial, neurocognitive and skeletal development caused by mutations in TGFBR1 or TGFBR2. Nat Genet. 2005;37:275-81.

9. Pannu H, Fadulu VT, Chang J, et al. Mutations in transforming growth factor-beta receptor type II cause familial thoracic aortic aneurysms and dissections. Circulation. 2005;112:513-20.

10. Roberts WC, Ko JM. Frequency by decades of unicuspid, bicuspid, and tricuspid aortic valves in adults having isolated aortic valve replacement for aortic stenosis, with or without associated aortic regurgitation. Circulation. 2005;111:920-5.

11. Verloes A, Sakalihasan N, Koulischer L, Limet R. Aneurysms of the abdominal aorta: familial and genetic aspects in three hundred thirteen pedigrees. $J$ Vasc Surg. 1995;21:646-55.

12. Gadson PF Jr, Dalton ML, Patterson E, et al. Differential response of mesodermand neural crest-derived smooth muscle to TGF-beta1: regulation of c-myb and alpha1 (I) procollagen genes. Exp Cell Res. 1997;230:169-80.

13. Dalton ML, Gadson PF Jr, Wrenn RW, Rosenquist TH. Homocysteine signa cascade: production of phospholipids, activation of protein kinase $\mathrm{C}$, and the induction of c-fos and c-myb in smooth muscle cells. FASEB J. 1997;11:703-11.

14. Thieszen SL, Dalton M, Gadson PF, et al. Embryonic lineage of vascular smooth muscle cells determines responses to collagen matrices and integrin receptor expression. Exp Cell Res. 1996;227:135-45.

15. Wolinsky H, Glagov S. Comparison of abdominal and thoracic aortic medial structure in mammals. Deviation of man from the usual pattern. Circ Res. 1969; 25:677-86.

16. Wolinsky H. Comparison of medial growth of human thoracic and abdominal aortas. Circ Res. 1970;27:531-8.

17. Halloran BG, Davis VA, McManus BM, et al. Localization of aortic disease is associated with intrinsic differences in aortic structure. J Surg Res. 1995;59:17-22.

18. Vorp DA, Raghavan ML, Muluk SC, et al. Wall strength and stiffness of aneurysmal and nonaneurysmal abdominal aorta. Ann N Y Acad Sci. 1996;800:274-6.

19. Fillinger MF, Marra SP, Raghavan ML, Kennedy FE. Prediction of rupture risk in abdominal aortic aneurysm during observation: wall stress versus diameter. J Vasc Surg. 2003;37:724-32.

20. Groenink M, Langerak SE, Vanbavel E, et al. The influence of aging and aortic stiffness on permanent dilation and breaking stress of the thoracic descending aorta. Cardiovasc Res. 1999;43:471-80.

21. Raghavan ML, Webster MW, Vorp DA. Ex vivo biomechanical behavior of abdominal aortic aneurysm: assessment using a new mathematical model. Ann Biomed Eng. 1996;24:573-82.

22. Vorp DA, Schiro BJ, Ehrlich MP, et al. Effect of aneurysm on the tensile strength and biomechanical behavior of the ascending thoracic aorta. Ann Thorac Surg. 2003;75:1210-4

23. Flamant M, Placier S, Dubroca C, et al. Role of matrix metalloproteinases in early hypertensive vascular remodeling. Hypertension. 2007;50:212-8.

24. He CM, Roach MR. The composition and mechanical properties of abdominal aortic aneurysms. J Vasc Surg. 1994;20:6-13.

25. Strong JP, Malcom GT, McMahan CA, et al. Prevalence and extent of atherosclerosis in adolescents and young adults: implications for prevention from the Pathobiological Determinants of Atherosclerosis in Youth Study. JAMA. 1999;281: 727-35.

26. Homma S, Troxclair DA, Zieske AW, et al. Histological topographical comparisons of atherosclerosis progression in juveniles and young adults. Atherosclerosis. 2008;197:791-8.

27. Galis ZS, Khatri JJ. Matrix metalloproteinases in vascular remodeling and atherogenesis: the good, the bad, and the ugly. Circ Res. 2002;90:251-62. 
28. Aimes RT, Quigley JP. Matrix metalloproteinase-2 is an interstitial collagenase. Inhibitor-free enzyme catalyzes the cleavage of collagen fibrils and soluble native type I collagen generating the specific 3/4- and 1/4-length fragments. J Biol Chem. 1995;270:5872-6.

29. Lesauskaite V, Tanganelli P, Sassi C, et al. Smooth muscle cells of the media in the dilatative pathology of ascending thoracic aorta: morphology, immunoreactivity for osteopontin, matrix metalloproteinases, and their inhibitors. Hum Pathol. 2001;32:1003-11.

30. Sinha I, Bethi S, Cronin P, et al. A biologic basis for asymmetric growth in descending thoracic aortic aneurysms: a role for matrix metalloproteinase 9 and 2 . J Vasc Surg. 2006;43:342-8.

31. Schmoker JD, McPartland KJ, Fellinger EK, et al. Matrix metalloproteinase and tissue inhibitor expression in atherosclerotic and nonatherosclerotic thoracic aortic aneurysms. J Thorac Cardiovasc Surg. 2007;133:155-61.

32. McNulty M, Spiers P, McGovern E, Feely J. Aging is associated with increased matrix metalloproteinase-2 activity in the human aorta. Am J Hypertens. 2005; 18:504-9.

33. Barbour JR, Stroud RE, Lowry AS, et al. Temporal disparity in the induction of matrix metalloproteinases and tissue inhibitors of metalloproteinases after thoracic aortic aneurysm formation. J Thorac Cardiovasc Surg. 2006;132:788-95.

34. Crowther M, Goodall S, Jones JL, et al. Increased matrix metalloproteinase 2 expression in vascular smooth muscle cells cultured from abdominal aortic aneurysms. J Vasc Surg. 2000;32:575-83.

35. Freestone T, Turner RJ, Coady A, et al. Inflammation and matrix metalloproteinases in the enlarging abdominal aortic aneurysm. Arterioscler Thromb Vasc Biol. 1995;15:1145-51.

36. Longo GM, Xiong W, Greiner TC, et al. Matrix metalloproteinases 2 and 9 work in concert to produce aortic aneurysms. J Clin Invest. 2002;110:625-32.

37. Jones JA, Barbour JR, Lowry AS, et al. Spatiotemporal expression and localization of matrix metalloproteinas-9 in a murine model of thoracic aortic aneurysm. $J$ Vasc Surg. 2006;44:1314-21.

38. Tamarina NA, McMillan WD, Shively VP, Pearce WH. Expression of matrix metalloproteinases and their inhibitors in aneurysms and normal aorta. Surgery. 1997; 122:264-72.

39. McMillan WD, Tamarina NA, Cipollone M, et al. Size matters: the relationship between MMP-9 expression and aortic diameter. Circulation. 1997;96:2228-32.
40. Xiong W, Knispel R, Mactaggart J, Baxter BT. Effects of tissue inhibitor of metalloproteinase 2 deficiency on aneurysm formation. J Vasc Surg. 2006;44: 1061-6.

41. Koullias GJ, Ravichandran P, Korkolis DP, et al. Increased tissue microarray matrix metalloproteinase expression favors proteolysis in thoracic aortic aneurysms and dissections. Ann Thorac Surg. 2004;78:2106-11.

42. Roberts AB, Sporn MB, Assoian RK, et al. Transforming growth factor type beta: rapid induction of fibrosis and angiogenesis in vivo and stimulation of collagen formation in vitro. Proc Natl Acad Sci U S A. 1986;83:4167-71.

43. Absi TS, Sundt TM 3rd, Tung WS, et al. Altered patterns of gene expression distinguishing ascending aortic aneurysms from abdominal aortic aneurysms: complementary DNA expression profiling in the molecular characterization of aortic disease. J Thorac Cardiovasc Surg. 2003;126:344-57.

44. Middleton RK, Lloyd GM, Bown MJ, et al. The pro-inflammatory and chemotactic cytokine microenvironment of the abdominal aortic aneurysm wall: a protein array study. J Vasc Surg. 2007;45:574-80.

45. Kusano K, Miyaura C, Inada M, et al. Regulation of matrix metalloproteinases (MMP-2, -3, -9, and -13) by interleukin-1 and interleukin-6 in mouse calvaria: association of MMP induction with bone resorption. Endocrinology. 1998;139: 1338-45.

46. Tang PC, Yakimov AO, Teesdale MA, et al. Transmural inflammation by interferon-gamma-producing $\mathrm{T}$ cells correlates with outward vascular remodeling and intimal expansion of ascending thoracic aortic aneurysms. FASEB J. 2005; 19:1528-30.

47. Galle C, Schandene L, Stordeur P, et al. Predominance of type 1 CD4+T cells in human abdominal aortic aneurysm. Clin Exp Immunol. 2005;142:519-27.

48. Schonbeck U, Sukhova GK, Gerdes N, Libby P. T(H)2 predominant immune responses prevail in human abdominal aortic aneurysm. Am J Pathol. 2002;161: 499-506.

49. Chung AW, Au Yeung K, Sandor GG, et al. Loss of elastic fiber integrity and reduction of vascular smooth muscle contraction resulting from the upregulated activities of matrix metalloproteinase-2 and -9 in the thoracic aortic aneurysm in Marfan syndrome. Circ Res. 2007;101:512-22.

50. Dai J, Losy F, Guinault AM, et al. Overexpression of transforming growth factorbeta1 stabilizes already-formed aortic aneurysms: a first approach to induction of functional healing by endovascular gene therapy. Circulation. 2005;112:1008-15. 